\title{
Sphingomonas paucimobilis: Un phénotype de multi-résistance inhabituel chez un probable pathogène émergent
}

\section{Sphiongomonas paucimobilis: A multidrug resistance pattern in emerging like bacterial pathogen}

MAKANERA $\mathrm{A}^{1,2}$, Condé $\mathrm{M}^{2}$, DiALlO $\mathrm{MA}^{2}$, Condé $\mathrm{M}^{2}$, Camara $\mathrm{D}^{2}$

1-Université Gamal Abdel Nasser de Conakry, Rép. GUINEE et Laboratoire biomédical de l'Hôpital de l'Amitié Sino-Guinéenne, Kipé, Cité des Médecins, Commune Ratoma 30 BP : 710 Conakry, GUINEE

2-Service de Laboratoire biomédical de l'Hôpital de l'Amitié Sino-Guinéenne, Kipé, Cité des Médecins, Commune Ratoma 30 BP : 710 Conakry, GUINEE

Correspondance : Dr Abdoulaye MAKANERA Email: abdmak@yahoo.fr

\section{RESUME}

Introduction: La multi-résistance aux antibiotiques devient un phénomène inquiétant chez des bactéries pathogènes et pathogènes émergentes. Objectif: L'objectif de ce travail était de décrire un phénotype de multi-résistance aux antibiotiques chez une souche de Sphingomonas paucimobilis. Patiente et méthodes: Une patiente de 55 ans a été reçue le 29 Novembre 2017 à l'Hôpital de l'Amitié Sino-Guinéenne (Kipé/Conakry) pour des examens cytobactériologiques des urines. L'identification bactérienne et l'antibiogramme ont été faites à l'automate Vitek2 compact 15 (Biomérieux, Marcy l'Etoile, France). Résultats : L'identification bactérienne a révélé la présence de Sphingomonas paucimobilis. Les antibiogrammes réalisés avec les cartes AST-N 233 et AST-XN05 ont montré une résistance à tous les antibiotiques testés avec des CMI élevées, à l'exception des tétracyclines et du chloramphénicol. Conclusion : Nos résultats montrent que la souche de Sphingomonas paucimobilis étudiée pourrait être considérée comme une bactérie pathogène émergente présentant un phénotype de multi-résistance à différentes familles d'antibiotiques.

Mots clés : Sphingomonas paucimobilis, antibiotiques, multi-résistance, Kipé/Conakry.

\footnotetext{
ABSTRACT:

Introduction: Bacterial multidrug resistance is becoming a worldwide health problem in pathogenic bacteria, and emerging bacterial pathogen. Objective: The aim of this study was to describe a multidrug resistance pattern in Sphingomonas paucimobilis, isolated from urine sample. Patient and methods: A 55 years old woman was received at the Hôpital de l'Amitié Sino-Guinéenne of Kipé/Conakry for biological analysis at the November 29th, 2017. The bacterial identification and antibiograms were processed on Vitek 2 Compact 15 (Biomérieux, Marcy l'Etoile, France). Results: The bacterial isolate was identified as Sphingomonas paucimobilis. The antibiograms using AST-N 233 and AST-XN05 cards showed that this isolate was resistant to all
}

antibiotics tested with high MICs, except for tetracyclines and chloramphenicol to which, this isolate was susceptible. Conclusion: Our findings showed that this isolate of Sphingomonas paucimobils must be considered as an emerging bacterial pathogen showing a multidrug resistance pattern to nearly all antibiotic families.

Keywords : Sphingomonas paucimobilis, antibiotics, multiresistance, Kipé/Conakry

\section{INTRODUCTION:}

La multi-résistance aux antibiotiques devient un phénomène inquiétant dans le monde aussi bien chez les bactéries habituellement pathogènes que chez des pathogènes émergentes autre fois considérées comme des germes de l'environnement comme Sphingomonas paucimobilis [1]. En effet, Sphingomonas paucimobilis anciennement appelé Pseudomonas paucimobilis est une bactérie se présentant sous la forme de bacilles à Gram négatif d'une large distribution dans l'environnement notamment dans l'eau et le sol. Il s'agit d'une espèce bactérienne caractérisée par la production de pigment jaune (d'où la coloration jaune de ses colonies en milieux gélosés); elle est non fermentaire et pourvue d'un seul flagelle polaire lui permettant une faible mobilité, d'où l'appellation paucimobilis. Souvent considérée par le passé comme une bactérie de moindre importance médicale, Sphingomonas paucimobilis devient de plus en plus fréquemment isolée des infections communautaires et hospitalières $[1,2]$. Cependant, ces infections sont décrites de façon sporadique. En effet, les premières descriptions de $S$. paucimobilis en pathologie humaine ont été faites en 1979 [1]. Depuis cette date, les infections à $S$. paucimobilis ont été rapportées dans diverses sécrétions biologiques potentiellement pathologiques $[1,2]$. Des infections sévères, comme les bactériémies/septicémies à $S$. paucimobilis ont été rapportées par de nombreux auteurs [2,3,4,5]. Cependant, ces infections ne semblent encore associées qu'à de très rares cas de décès [2]. L'émergence de souches de $S$. paucimobilis multirésistantes aux antibiotiques avec des profils de 
sensibilité variant d'une souche à l'autre a été rapportée par de nombreuses études $[1,3,5,6]$. Cette situation devient plus inquiétante lors que la souche bactérienne montre un profil de multi-résistance à toutes les familles d'antibiotiques (bêta-lactamines, aminosides, fluoroquinolones, nitrofurantoine, sulfamides et leurs associations). L'apparition de telles souches bactériennes pourraient être à l'origine de nombreux cas d'échecs thérapeutiques antibactériens. En 2017, Makanéra et al., [1] ont montré dans leur étude que les infections à $S$. paucimobilis semblent devenir, un problème de santé publique en Guinée. L'objectif de cette présente étude était de décrire un phénotype inhabituel de multi-résistance à presque toutes les familles d'antibiotiques testés chez $S$. paucimobilis.

\section{PATIENTE ET METHODES:}

Description du Cas: Une patiente âgée de 55 ans et de profession ménagère a été reçue le 29 Novembre 2017 au Laboratoire biomédical de l'HASIGUI en provenance du Service de Chirurgie viscérale pour des examens biologiques. Les examens demandés étaient l'analyse cytobactériologique des urines, la sérologie du virus de l'immunodéficience humaine (VIH), l'hémogramme, la détermination de l'activité enzymatique des transaminases (GPT: Transaminases glutamique pyruvique ou ALAT: Alanine aminotransférases; GOT: Transaminase glutamique oxaloacétique ou ASAT: Aspartate aminotransférase), et le dosage de la bilirubine sérique: bilirubine totale (Bil $\mathrm{T}$ ) et bilirubine directe (Bil D). L'analyse bactériologique a été faite sur un volume de $50 \mathrm{~mL}$ d'urines de la patiente recueillies dans un flacon stérile. Le prélèvement d'urines a été réalisé dans les toilettes de la salle de prélèvement du laboratoire biomédical. Après avoir donné des instructions à la patiente: lavage vulvo-vaginal avec du savon et recueil d'urines au milieu du jet, suivi du transport en quelques minutes dans la salle des prélèvements où sont collectés, numérotés et enregistrés tous les échantillons. Les examens cytobactériologiques ont été entamés dans les 15 minutes suivantes. Le sang veineux prélevé chez la patiente a servi pour les examens biochimiques, immuno-sérologiques et hématologiques. L'échantillon d'urines a été soumis à l'examen cytobactériologique à l'état frais par observation au microscope optique (Microscope XS-213, Nanjing BW Optics Co., Ltd., Jiangsu, China) suivi de la coloration de Gram des lames porte-objet examinés. Un kit de coloration des bactéries par la méthode de Gram-Hücker (RAL Diagnostics, Martillac, France) a été utilisé. L'échantillon a été ensuite mis en culture sur différents milieux gélosés : gélose Columbia au sang de mouton 5\% (Liofilchem, Roseto D.A., Italy), gélose nutritive (Liofilchem, Roseto D.A., Italy), gélose Mac Conkey (Biomérieux, Marcy l'Etoile, France) et CLED (Biomérieux, Marcy
l'Etoile, France). L'incubation a été faite pendant 18 heures à l'étuve GRP 9080 (Sumsung Laboratory Instrument CO., Ltd, Shanghai, China). Des colonies bactériennes uniformes isolées des cultures ont été colorées par la méthode de Gram afin de vérifier leur pureté, étape clé précédant les analyses à l'automate Vitek 2 Compact 15 (Biomérieux, Marcy l'Etoile, France). L'identification bactérienne, les antibiogrammes et la détermination des concentrations minimales inhibitrices (CMI) ont été réalisés à l'aide de l'automate Vitek 2 compact 15 (Biomérieux, Marcy l'Etoile, France). Les cartes Vitek $2 \mathrm{GN}$ ont servi pour l'identification et les cartes Vitek 2 AST-N 233 (Biomérieux, Marcy l'Etoile, France) ainsi que celles AST-XN05 (Biomérieux, Marcy l'Etoile, France) ont servi pour les antibiogrammes et la détermination des CMI, en suivant les instructions du fabricant (Biomérieux, Marcy l'Etoile, France). Les cartes Vitek 2 GN prêtes à l'emploi contiennent 64 puits correspondant à 64 réactions permettant d'identifier des bacilles à Gram négatif (Enterobacteriaceae, Non-Enterobacteriaceae et germes hautement pathogènes). Les Cartes Vitek 2 AST-N 233 prêtes à l'emploi contenant 18 antibiotiques et associations d'antibiotiques appartenant à différentes familles sont destinés à la fois aux bactéries à Gram négatif fermentaires et non fermentaires. Ces antibiotiques sont: l'ampicilline, l'association amoxicilline/acide clavulanique, la ticarcilline, l'association pipéracilline/Tazobactam, la céphalotine, la céfoxitine, le céfotaxime, la ceftazidime, l'imipénème, l'ertapénème, l'amikacine, la gentamicine, la tobramycine, l'acide nalidixique, la ciprofloxacine, l'ofloxacine, la nitrofurantoine, et le triméthoprime/Sulfaméthoxazole. Les résultats des antibiotiques non requis sont éliminés automatiquement par le Vitek 2 compact 15 (Biomérieux, Marcy l'Etoile, France). Les cartes Vitek 2 AST-XN05 (Biomérieux, Marcy l'Etoile, France) destinés aux bacilles à Gram négatif multirésistantes ont été par la suite utilisées afin de détecter d'éventuels antibiotiques actifs sur la souche de $S$. paucimobilis analysée et de donner une possibilité d'antibiothérapie adaptée à la souche. Les 18 antibiotiques et associations d'antibiotiques utilisés dans les cartes Vitek 2 ASTXN05 (Biomérieux, Marcy l'Etoile, France) étaient: la pipéracilline, l'association ampicilline/sulbactam, la céfuroxime, la céfuroxime axétil, la céfixime, la ceftriaxone, le céfépime, l'aztréonam, le méropénème, la lévofloxacine, la moxifloxacine, la minocycline, la tétracycline, la tigécycline, le Chloramphénicol, la triméthoprime, la colistine et l'association ticarcilline/acide clavulanique. Dans le système Vitek 2 (Biomérieux, Marcy l'Etoile, France) compact, les résultats des antibiogrammes et des CMI ont été déterminés selon les critères et normes 
d'interprétation de Clinical and Laboratory Standards Institute (CLSI). Le logiciel Advanced Expert System (AES) compare les CMI de l'instrument et l'identité du germe identifié aux phénotypes standard de ce germe (CLSI + Natural Resistance). L'AES communique les résultats obtenus sous forme de rapports récapitulatifs et de propositions. En fonction des germes identifiés, les résultats des antibiotiques jugés non requis sont supprimés dans le rapport des résultats de l'antibiogramme déterminés par le système Vitek 2 compact 15 (Biomérieux, Marcy l'Etoile, France). Ainsi, pour les cartes AST-N 233, les résultats de sensibilité de l'ertapénème sont toujours supprimés pour $S$. paucimobilis alors que ceux de la colistine et l'association ticarcilline/acide clavulanique sont supprimés par l'automate pour les AST XN05. La sérologie VIH a été faite parallèlement à l'aide des Kits spécifiques (HIV Determine et HIV Combo, Alere Medical Co., Ltd. Matsuhidai, Japan). En cas de positivité, la confirmation du résultat de la sérologie VIH serait réalisée par la technique immuno-enzymatique à l'aide de l'automate miniVidas utilisant des kits VIDAS HIV Duo Ultra (Biomérieux, Marcy l'Etoile, France). L'hémogramme a été réalisé à l'automate Mindray BC 2800 (Mindray, Shenzen, China). La mesure de l'activité des transaminases et le dosage de la bilirubine ont été faits à l'automate Mindray BS 200 (Mindray, Shenzen, China).

RESULTATS: Les résultats de l'examen cytobactériologique des urines ont montré une leucocyturie (purée leucocytaire, c'est-à-dire des leucocytes innombrables). Les cultures bactériennes ont révélé la présence de colonies jaunâtres sur milieux gélosé Columbia au sang de mouton 5\% (Figure 1). Les colorations de Gram de colonies bactériennes isolées après culture ont permis d'observer des bacilles à Gram négatif.

L'identification et l'antibiogramme des colonies bactériennes isolées de l'échantillon d'urines ont montré la présence d'une souche de $S$. paucimobilis multi-résistante à toutes les familles d'antibiotiques testés avec des CMI élevées (Tableau I). Les antibiotiques suivants : l'ertapénème (utilisé dans les cartes AST-N233), la colistine et l'association ticarcilline/acide clavulanique et la colistine (utilisés dans les cartes AST-XN05) ont été éliminé automate Vitek 2 Compact 15 (Biomérieux, Marcy l'Etoile, France) lors de l'analyse et sont considérés non requis pour l'espèce $S$. paucimobilis. La sérologie VIH a montré que la patiente était séronégative pour le VIH. Les résultats de l'hémogramme ont montré que la plupart des paramètres étudiés étaient dans les limites des valeurs de référence. En revanche, la concentration corpusculaire moyenne des hématies et la teneur corpusculaire en hémoglobine étaient, respectivement, inferieure et légèrement inférieure aux valeurs de référence correspondantes alors que la valeur des plaquettes était légèrement supérieure à la valeur normale (Tableau II). D'autre part, pour les examens biochimiques, les valeurs des transaminases étaient dans les limites des valeurs de référence alors que celles de la bilirubine totale et directe étaient supérieures aux valeurs de référence. DISCUSSION:De nombreuses études ont montré la présence de $S$. paucimobilis dans diverses fluides biologiques tels que le sang au cours des bactériémies, le liquide céphalorachidien, les urines, différentes suppurations dont les arthrites septiques, les ostéomyélites, le pus intra abdominal, les prélèvements pulmonaires suppurés, les péritonites au cours des dialyses, et les endophtalmies post-chirurgicales [1,4,6]. En 2017, Makanéra et $a l$., ont décrit dans une même étude, l'isolement de S. paucimobilis dans des secrétions vaginales, du sperme, du liquide pleural, du liquide d'ascite, des selles diarrhéiques, des urines [1].

Phénotype de résistance de $S$. paucimobilis aux antibiotiques: De nombreux auteurs tels que Bhatia et Tomar (2016) cités en 2017 par Makanéra et al., avaient rapporté l'isolement de $S$. paucimobilis dans différentes sécrétions biologiques en lui donnant un caractère de bactérie pathogène émergente [1]. En effet, l'incidence des infections à $S$. paucimobilis semble avoir augmenté ces deux dernières décennies à travers le monde. Cette tendance à l'augmentation pourrait continuer. Ce constat semble en rapport avec la définition des maladies infectieuses émergentes de Centers for Diseases Control (CDC) and Prevention d'Atlanta (USA) selon laquelle: «Les maladies infectieuses émergentes sont des maladies infectieuses dont l'incidence chez l'Homme a augmenté au cours des deux dernières décennies ou qui menace d'augmenter dans un avenir proche» [7]. L'acquisition d'un phénotype de multi-résistance pourrait être un facteur important d'évolution de l'écologie bactérienne. Woolhouse et GowtageSequeria en 2005, dans leur étude sur les mécanismes associés à l'émergence ou à la réémergences des germes pathogènes infectant l'Homme, ont établi une classification de ces mécanismes en dix catégories [8]. L’ordre de classification est fonction de l'importance du nombre d'espèces pathogènes associées à ces mécanismes. L'évolution des pathogènes due à la résistance aux antimicrobiens ou à l'augmentation de leur virulence occupe le $5^{\text {ème }}$ rang de cette classification. Cependant, de rares études ont porté sur les profils de sensibilité de S. paucimobilis aux antibiotiques [1-3,5,9]. Actuellement, il n'existe aucune description des mécanismes de résistance aux antibiotiques chez $S$. paucimobilis. D'autre part, il existe très peu d'études sur les concentrations minimales inhibitrices de $S$. paucimobilis aux antibiotiques [1,2]. Par ailleurs, il n'existe à notre connaissance aucune étude sur $S$. paucimobilis ayant déterminé des résistances 
naturelles aux antibiotiques chez cette espèce. Il faut aussi remarquer que de nombreuses souches de S. paucimobilis montrant une sensibilité totale à tous les antibiotiques dans les cartes AST-N 233 (citées plus haut) ont été isolées dans notre laboratoire (Makanéra et al., 2017). Cette présente étude décrit une souche de $S$. paucimobilis montrant des cas de multi-résistance encore jamais décrit, à notre connaissance dans la littérature.

- Résistance aux bêta-lactamines: Les résultats de l'antibiogramme obtenus avec les cartes Vitek 2 AST-N 233 ont montré que la souche de $S$. paucimobilis que nous avons isolée était résistante à l'ampicilline, l'association amoxicilline/acide clavulanique, la ticarcilline, l'association pipéracilline/tazobactam, la céphalotine, la céfoxitine, le céfotaxime, la ceftazidime, et l'imipénème (Tableau I). La résistance de cette souche à l'imipénème et à l'association pipéracilline/Tazobactam est semblable à celle observée par Kumar et al., (2015) qui ont décrit une souche de $S$. paucimobilis isolée d'une infection du tractus urinaire, résistante (entre autres) à ces antibiotiques [9]. Cette résistance est également semblable à celle d'une souche de $S$. paucimobilis isolée à l'Hôpital de l'Amitié Sino-Guinéenne (Conakry) à partir d'un liquide d'ascite chez une femme âgée de 83 ans [1]. En effet, cette dernière était la seule souche ayant montré une résistance à l'imipénème parmi les seize échantillons de $S$. paucimobilis décrits par ces auteurs. En revanche, ces résultats sont différents de ceux observés pour la majorité des autres souches décrites par ces auteurs au cours d'une même étude et qui présentaient une sensibilité totale à l'imipénème avec des CMI très bas [1]. Par ailleurs, Lugito et $a l$, ont décrit une souche de $S$. paucimobilis isolée $\mathrm{d}$ 'hémoculture et sensible à l'imipenème [2]. Ces auteurs n'ont pas cependant donné d'information sur la sensibilité de leur souche à l'association pipéracilline/tazobactam [2]. De même Bayram et al., en 2013, avaient décrit la sensibilité de leurs souches de $S$. paucimobilis à l'imipenème [5]. Egalement la majorité de leurs souches étaient sensibles au céfotaxime, seuls 20,8\% (5/24) étaient résistantes à cette molécule, ce qui est semblable aux données rapportées par Makanéra et al. en 2017 [1]. Pour l'ampicilline, Bayram et al. ont trouvé que seulement $12,5 \% \quad(3 / 24)$ des souches étaient résistantes à cette molécule en 2013 [5], alors que Makanéra et $a l$., avaient trouvé que 56,25\% (9/16) des souches de $S$. paucimobilis étaient résistantes à cette dernière [1]. Ces auteurs avaient également rapporté que $87,5 \%(14 / 16)$ des souches de $S$. paucimobilis étaient sensibles à l'association amoxicilline/acide clavulanique, alors que 50\% (8/16) étaient résistantes à la ceftazidime [1]. Lugito et $a l$, avaient également décrit la résistance de leur souche à l'ampicilline, la ceftazidime et la ceftriaxone [2]. Le fait que la souche de $S$. paucimobilis soit résistante à toutes les bêtalactamines et inhibiteur de bêta-lactamases utilisées dans les cartes Vitek 2 AST-N 233 nous avait conduit à réaliser l'antibiogramme de cette souche avec les cartes Vitek 2 AST-XN05 afin de déterminer d'éventuels antibiotiques actifs sur la souche isolée et assurer une antibiothérapie adaptée à l'infection urinaire chez la patiente. Les résultats obtenus ont montré une résistance totale de cette souche bactérienne à l'association ampicilline/sulbactam, la céfuroxime, la céfuroxime axétil, la céfixime, la ceftriaxone, le céfépime, l'aztréonam, et le méropénème (Tableau I). En revanche, cette souche avait par contre montré une sensibilité intermédiaire à la pipéracilline $(\mathrm{CMI}=32 \mu \mathrm{g} / \mathrm{ml})$. Ces résultats sont en partie, différents de ceux de Lin et al., qui en 2010, ont montré que la majorité de leurs souches, respectivement $81,3 \%(13 / 16)$ et $68,8 \%(11 / 16)$ étaient sensibles à l'association ampicilline/sulbactam et à la pipéracilline [3]. Nos résultats sont également différents de ceux de Bayram et al., qui ont trouvé en 2013 que seulement $8,3 \%(2 / 24)$ de leurs souches étaient résistantes à la céfuroxime d'une part et d'autre part à la pipéracilline [5]. Hsueh et $a l$., ont montré dans leur étude en 1998 sur 11 souches cliniques et 3 souches environnementales, une sensibilité à la céfuroxime variable selon les souches [1]. Dans le présent travail, la souche de $S$. paucimobilis étudiée a montré une résistance de haut niveau à la céfuroxime $(\mathrm{CMI} \geq 64 \mu \mathrm{g} / \mathrm{ml})$. Cette résistance à la céfuroxime de la souche de $S$. paucimobilis est semblable à celle rapportée par certains auteurs $[2,4]$. A notre connaissance, il existe très peu de données dans la littérature sur la sensibilité de $S$. paucimobilis à la céfuroxime axétil. Les données rapportées par Özdzmir et al., en 2011 ont montré que leur souche de $S$. paucimobilis était sensible la céfuroxime axétil, contrairement aux résultats de cette présente étude. Le présent travail serait alors l'une des premières descriptions de la résistance de $S$. paucimobilis à cette molécule.La résistance de la souche de S. paucimobilis à la ceftriaxone dans le présent travail est semblable à celle décrite en 2011 par Özedemir et al., chez une souche de $S$. paucimobilis isolée au cours d'une bactériémie chez un enfant atteint du syndrome de Down [4]. Cette observation est différente de celle rapportée par d'autres auteurs [6]. En effet, en 2011, Krishna et $a l$, ont rapporté dans leur étude que Smallay et $a l$., avaient observé la sensibilité à la ceftriaxone des 20 souches de $S$. paucimobilis rapportées dans leur étude [6]. Il faut noter que dans la littérature, la sensibilité de $S$. paucimobilis à la ceftriaxone a été rarement testée contrairement aux autres céphalosporines de troisième génération $(\mathrm{C} 3 \mathrm{G})$, le céfotaxime et la ceftazidime. Cependant, la sensibilité ou la résistance d'une souche bactérienne à deux de ces $\mathrm{C} 3 \mathrm{G}$ pourrait souvent être extrapolée 
à la troisième molécule. En Guinée, l'émergence de souches bactériennes résistantes à la ceftriaxone comme la souche de $S$. paucimobilis dans le présent travail est un véritable problème de santé publique. En effet, la ceftriaxone est de loin la C3G la plus utilisée dans le pays par les cliniciens depuis de nombreuses années. L'utilisation anarchique de cette molécule est une réalité à tous les niveaux de la pyramide sanitaire guinéenne. Ainsi, la lutte contre ce phénomène d'utilisation anarchique et abusive de la ceftriaxone ainsi que d'autres molécules antibactériennes doit être menée auprès des cliniciens et des populations responsables d'automédication. La résistance de $S$. paucimobilis à la céfépime de cette présente étude est semblable à celle décrite par certains auteurs [2,4]. En revanche, ces observations sont différentes de celles faites par Lin et $a l$., en 2010 pour la majorité $(68,8 \%=11 / 16)$ de leurs souches de $S$. paucimobilis qui étaient sensibles à cette molécule. Des données sur la sensibilité de $S$. paucimobilis à la céfixime semble absente (ou rare) dans la littérature. Ainsi, la résistance de $S$. paucimobilis à la céfixime observée dans le présent travail pourrait être l'une des premières données rapportées dans la littérature. En 2016, Lugito et al., ont décrit une souche de $S$. paucimobilis isolée d'une bactériémie chez un patient immunodéprimé [2]. Cette souche associée à l'un des rares cas d'infection létale à $S$. paucimobilis était totalement résistante à l'aztréonam comme dans le présent travail. En revanche, nos résultats sont différents de ceux observés en 2008 par Seo et al., rapportés par Makanéra et al., en 2017, qui ont trouvé une sensibilité totale de leur souche de $S$. paucimobilis à l'aztréonam [1]. La souche de $S$. paucimobilis décrite par Lugito et al., en 2016 avait une sensibilité intermédiaire au méropénème, contrairement à celle rapportée dans le présent travail qui montrait une résistance de haut niveau $(\mathrm{CMI} \geq 64 \mu \mathrm{g} / \mathrm{ml})$ à cette molécule [2]. Nos résultats sont aussi différents de ceux de Nandy et al., cités par Makanéra et al., en 2017, qui avaient trouvé en 2013 que toutes les deux souches de $S$. paucimobilis qu'ils ont étudiées étaient sensibles au méropénème [1]. Enfin, nos résultats sont également différents de ceux trouvés par Özdemir et al., (2011) qui ont décrit la sensibilité au méropénème d'une souche de $S$. paucimobilis isolée d'une bactériémie chez un enfant atteint du syndrome de Down [4]. La souche de $S$. paucimobilis étudiée dans le présent travail a montré une résistance totale à toutes les bêtalactamines testées, à l'exception de la pipéracilline à laquelle cette souche a montré une résistance de bas niveau ou sensibilité intermédiaire (Tableau I). Ces données pourraient suggérer une possibilité de production des bata-lactamases. Ces observations sont similaires à celles déjà faite par Smalley et $a l$., qui ont été cités par Lin et al. en 2010. Ces auteurs ont soutenu que toutes les souches de $S$. paucimobilis étudiées étaient résistantes à la majorité des pénicillines et aux céphalosporines.

- Résistances aux aminosides : L'antibiogramme réalisé sur notre souche de $S$. paucimobilis à l'aide des cartes Vitek 2 AST-N 233 a montré qu'elle était résistante à l'amikacine, la gentamicine, et la tobramycine (Tableau I). Ces résultats sont en partie semblables (pour 1 cas) à ceux rapportés par Makanéra et al. (2017), qui ont trouvé une souche de $S$. paucimobilis isolée de liquide d'ascite chez une femme âgée de 83 ans et qui était résistante à l'amikacine, la gentamicine et la tobramycine. Cependant, les résultats de l'antibiogramme de cette souche sont différents de ceux trouvés en 2017 par Makanéra et al., pour la majorité $(93,75 \%)$ de leurs souches qui étaient sensibles à ces trois antibiotiques [1]. Lugito et al., en 2016 avaient également observé que leur souches de $S$. paucimobilis était résistante à l'amikacine et la gentamicine [2].

- Résistance aux fluoroquinolones: Les résultats de l'antibiogramme réalisé à l'aide des cartes Vitek 2 AST-N 233 ont montré dans le présent travail, que la souche de $S$. paucimobilis était résistante à l'acide nalidixique, la ciprofloxacine, l'ofloxacine (Tableau I). Ces résultats sont en partie semblables à ceux rapportés en 2016 par Lugito et al., (2016) qui ont trouvé leurs souches résistantes de à la ciprofloxacine et à la lévofloxacine [2]. En outre, Makanéra et $a l$., ont trouvé que la sensibilité de leurs souches de $S$. paucimobilis étaient de $62,6 \%$ $(10 / 16)$ à la ciprofloxacine, 56,25\% (9/25) à l'ofloxacine, alors que 68,75\% (11/16) étaient résistantes à l'acide nalidixique [1]. L'utilisation des cartes Vitek 2 AST-XN05 a révélé la résistance de la souche de $S$. paucimolis à la lévofloxacine et la moxifloxacine (Tableau I). Ces résultats sont différents de ceux rapportés par Kuo et al., qui ont montré une sensibilité de leurs souches à la lévofloxacine [1]. Nos résultats sont également différents de ceux d'Özdemir et al., qui avaient observé la sensibilité de leur souche de $S$. paucimobilis à la lévofloxacine [4]. La revue de la littérature montre qu'il existe très peu de données sur la sensibilité de $S$. paucimobilis à la moxifloxacine. Nos résultats concernant la résistance de $S$. paucimobilis à la moxifloxacine serait, en notre connaissance, l'une des premières descriptions de l'étude de la sensibilité de cette espèce bactérienne à la moxifloxacine.

- Résistance à la nitrofurantoine: L'antibiogramme réalisé sur notre souche de $S$. paucimobilis a montré qu'elle était hautement résistante à la nitrofurantoine avec une CMI très élevée $(\geq 512 \mu \mathrm{g} / \mathrm{ml})$ (Tableau I). Le degré de résistance observé à la nitrofurantoine chez cette souche dans le présent travail est largement supérieur à celui observé sur la souche de $S$. 
paucimobilis $\mathrm{N}^{\circ} 15$ décrite par Makanéra et al., (2017), isolée d'un liquide d'ascite prélevé chez une femme âgée de 83 ans [1]. En effet, cette souche avait une valeur de CMI $\geq 128 \mu \mathrm{g} / \mathrm{ml}$.

- Résistance aux sulfamides: Les résultats de l'antibiogramme obtenus à l'aide des cartes Vitek 2 AST-N233 ont montré que notre souche de $S$. paucimobilis était hautement résistante à l'association triméthoprime/sulfaméthoxazole (Tableau I). Ces résultats sont différents de ceux rapportés par Lugito et al. en 2016, par Kumar et $a l$., en 2015, qui ont trouvé que leurs souches de $S$. paucimobilis était sensibles à triméthoprime/sulfaméthoxazole [2,9]. Les résultats de l'antibiogramme réalisés à l'aide des cartes Vitek 2 AST-XN05 ont montré une résistance de haut niveau au triméthoprime, montrant ainsi que l'infection due à cette souche de $S$. paucimobilis la souche étudiée ne peut être traitée par cette classe d'antibiotique.

-Sensibilité aux tétracyclines : Les résultats de l'antibiogramme réalisé avec les cartes Vitek 2 AST-XN05 ont montré que la souche de $S$. paucimobilis étudiée était sensible à la minocycline, la tétracycline et la tigécycline (Tableau I). La sensibilité de la souche de S. paucimobilis étudiée dans le présent travail est semblable à celle rapporté par Almuzara et al., en 2011 qui ont observé une sensibilité de leur souche de $S$. paucimobilis à la tétracycline, la tigécycline et la minocycline [10]. La sensibilité de notre souche de $S$. paucimobilis à la tigécycline est semblable à celle observée en 2011 par Özdemir et al. D'autre part, Krishna et al., en 2011 ont rapporté que Smalley et $a l$., avaient montré que les 20 souches de $S$. paucimobilis décrites dans leur travail étaient sensibles à la tétracycline [6]. Almuzara et al., en 2011 ont décrit une souche de $S$. paucimobilis qui était à la fois sensible à la tétracycline, la tigécycline, la minocycline et la doxycyline [10]. Ces résultats sont très semblables aux nôtres, à l'exception de la doxycyline qui n'a pas été testée. Ces résultats ont ainsi permis de déterminer les antibiotiques actifs sur cette souche bactérienne, indiquant ainsi des possibilités d'une antibiothérapie adaptée de l'ITU chez la patiente par cette souche de $S$. paucimobilis multi-résistante aux antibiotiques.

-Sensibilité au chloramphénicol : Les résultats de l'antibiogramme réalisé en utilisant les cartes Vitek 2 AST-XN05 ont montré la souche de $S$. paucimobilis isolée d'ITU était sensible au chloramphénicol (Tableau I). Cependant, cette molécule a été retirée de la liste des antibiotiques utilisés en Guinée.De nombreuses données de la littérature montrent que la sensibilité des souches de $S$. paucimobilis est généralement variable [1,9]. En plus, il n'existe pas de méthode standard couramment utilisée par différents auteurs pour le choix des antibiotiques dans la réalisation des antibiogrammes chez $S$. paucimobilis $[2,3,6]$. Cette observation avait été faite préalablement par certains auteurs [2,3]. Dans la littérature, il n'existe pas encore de données (à notre connaissance) décrivant une résistance naturelle chez $S$. paucimobilis. Dans le présent travail, le phénotype de multi-résistance aux antibiotiques de la souche de $S$. paucimobilis pourrait être dû à l'utilisation abusive et anarchique des antibiotiques en Guinée, pouvant être à l'origine de l'émergence de ce nouveau phénotype de résistance observée chez cette souche bactérienne. En effet, la vente libre des antibiotiques (c'est-à-dire sans ordonnance médicale) et l'implication active des agents non professionnels dans le commerce de médicaments constitue une réalité en Guinée. A cela s'ajoute la circulation des médicaments d'origine douteuse et l'utilisation excessive des antibiotiques comme promoteurs de croissance des animaux ces dernières années dans le secteur de l'élevage. Enfin, la prescription abusive de la ceftriaxone par les professionnels même de la santé comme antibiotiques de première intention après diagnostic clinique d'infections bactériennes en dehors des résultats d'un antibiogramme est une réalité quotidienne chez les praticiens hospitaliers en Guinée. Toute la pyramide sanitaire est concernée par ce phénomène: postes de santé, centres de santé, hôpitaux préfectoraux, hôpitaux régionaux, centres médico-communaux et hôpitaux nationaux dans le secteur public, ainsi que les cabinets médicaux et cliniques privés présents dans tout le pays. Ce phénomène pourrait être à l'origine de nombreux cas d'échecs thérapeutiques antibactériens dans le pays. D'où la nécessité d'interpeler le gouvernement quant au contrôle des médicaments entrant dans le pays et le respect des normes de leur utilisation, mais également les professionnels du secteur de la santé à se conformer aux normes d'antibiothérapie en vigueur. La multirésistance de cette souche de $S$. paucimobilis observée au cours de la présente étude à 28 sur 33 molécules appartenant à différentes familles pourrait être due à l'association de plusieurs mécanismes de résistance bactérienne aux antibiotiques. D'où la nécessité d'envisager la recherche en biologie moléculaire (PCR, séquençage) des gènes de résistances à ces antibiotiques chez $S$. paucimobilis comme perspective. Dans les cas de multi-résistance aux antibiotiques, il y a nécessité d'élargir d'avantage le nombre d'antibiotiques testés pour la réalisation des antibiogrammes afin d'élargir aussi les possibilités thérapeutiques notamment par l'utilisation de cartes spéciales telle que la carte Vitek 2 AST XN05 destinées à des germes multi-résistants détectés par le système Vitek 2 Compact. Ce système capable de donner les résultats d'identification bactérienne et d'antibiogrammes en 8 à 16 heures permet la diligence du traitement chez le patient. Ceci rend 
ainsi l'utilisation du système Vitek 2 compact pour l'identification et l'antibiogramme plus performante que les systèmes API et la méthode de diffusion en gélose. Le système Vitek 2 compact permet l'utilisation d'un certain nombre de critères d'interprétation des résultats d'antibiogrammes. Les critères d'interprétation des antibiogrammes selon CLSI ont été utilisés dans ce présent travail car, ces critères sont de loin les plus utilisés par la majorité des travaux réalisés sur $S$. paucimobilis à travers le monde.

Conclusion : Nos résultats ont permis de montrer chez une souche de $S$. paucimobilis un phénotype de multi-résistance à presque toutes les familles d'antibiotiques testées, à l'exception des tétracyclines qui restent les seules molécules permettant ainsi de traiter l'IU chez notre patiente, le chloramphénicol étant retiré de la liste des antibiotiques actuellement utilisés en Guinée. L'ensemble de ces résultats ainsi que ceux préalablement publiés en Guinée et ailleurs dans le monde suggèrent que l'incidence des infections à $S$. paucimobilis menace d'augmenter dans un avenir proche, permettant ainsi de considérer cette bactérie comme un probable pathogène émergent capable d'acquérir un phénotype de résistance à presque toutes les familles d'antibiotiques couramment utilisées en Guinée.

\section{Conflit d'intérêt : aucun \\ Références}

1. Makanéra A, Condé M, Diallo MA, Condé M, Camara D, Barry AO, Diakité T. Profil de sensibilité des souches de Sphingomonas paucimobilis isolées de diverses secrétions biologiques à l'Hôpital de l'Amitié Sino-guinéenne kipé/Conakry (Guinée). REV. CAMES - Science de la vie, de la terre et agronomie, 2017 ; 5 (1) :14-20. 2. Lugito NPH, Nawangsih C, Kurniawan A. A lethal case of Sphingomonas paucimobilis bacteremia in an immunocompromised patient.
Case Report Infect. Dis. 2016; Article ID 3294639 , 4 pages.

3. Lin JN, Chung-Hsu L, Chenb YH, Lind HL, Huanga CK, Chena WF, et al. Sphingomonas paucimobilis bacteremia in Humans: 16 Case Reports and a Literature Review. J. Microbiol., Immunol. Infect., 2010; 43(1):35-42.

4. Özdemir M, Pekcan S, Demircili ME, Esenkaya F, Taşbent FE, Bahadır FB, et al. A Rare Cause of Bacteremia in a Pediatric Patient with Down Syndrome: Sphingomonas Paucimobilis. Internat. J. Med. Sces. 2011; 7:537-59.

5. Bayram N, Devrim I, Apa H, Gulfidan G, Turkydmaz HN, Gunay I. Sphingomonas paucimobilis infection in children: 24 case reports. Mediterr. J. Hematol. Infect. Dis., 2013; 5(1); e2013040. Doi: 10.4084/MJHID.2013.040.

6. Krishna S, Ciraj AM, Bairy I, Shobha KL. Sphingomonas paucimobilis urinary tract infection in renal transplant recipient: a rare case. Intern J. of Med and Public Health, 2011; 1:47-49.

7. CDC. Emerging infectious disease Wikipedia. 2003 . https://en.wikipedia.org/wiki/Emerging infectious disease, consulté le 25/08/2018

8. Woolhouser MEJ, Gowtage-Sequeria. Host range and Emerging and Re-emerging pathogens. Emerging Infectious Diseases, 2005; 11 (12): 184247. www.cdc.gov/eid.

9. Kumar M., Rawat V., Singh M. A., Bahugune D., Joshi S., Kumar U. Sphingomonas paucimobilis urinary tract infection in an immunocompetent patient: A case report. Intern. J. Med. Public Health, 2015; 5 (2):204.

10. Almuzara MN, Maria Isabel Baremotzallo MISE, Angela AMR, Vay CA. Comparative Activities of Tigecycline and Other Tetracyclines against Nonfermenting Gram-Negative Bacilli, Excluding Acinetobacter spp. Antimicrob. Agent Chamothér. 2011; 55 3961-63.

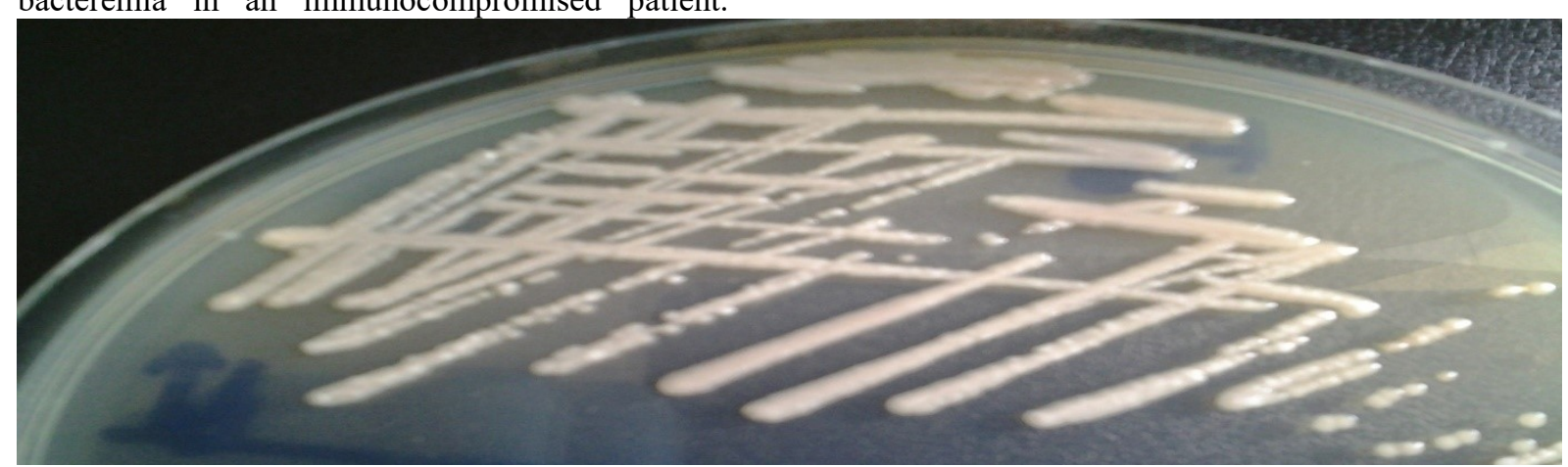

Figure 1 : Colonies de Sphingomonas paucimobilis sur gélose Columbia au sang de mouton 5\% après 24 heures d'incubation à $37^{\circ} \mathrm{C}$ à l'étuve GRP 9080 (Sumsung Laboratory Instrument CO., Ltd, Shanghai, China) 
Tableau I: Profil de Sensibilité de la souche de $S$. paucimobilis aux antibiotiques et leurs Concentrations minimales inhibitrices déterminés au Vitek 2 Compact 15 (Biomérieux, Marcy l'Etoile, France) avec les cartes AST-N 233 et AST-XN05

\begin{tabular}{|c|c|c|c|c|c|}
\hline \multicolumn{3}{|c|}{ Avec Carte AST-N 233} & \multicolumn{3}{|l|}{ Avec Carte AST-XN05 } \\
\hline Antibiotiques & Résistance & $\begin{array}{l}\text { CMI } \\
(\mu \mathrm{g} / \mathrm{ml})\end{array}$ & Antibiotiques & Catégorie & $\begin{array}{l}\text { CMI } \\
(\mu \mathrm{g} / \mathrm{ml})\end{array}$ \\
\hline Ampicilline & $\mathrm{R}$ & $\geq 32$ & & & \\
\hline $\begin{array}{l}\text { Amoxicilline/Ac. } \\
\text { clavulanique }\end{array}$ & $\mathrm{R}$ & $\geq 32$ & Ampicilline/sulbactam & $\mathrm{R}$ & $\geq 32$ \\
\hline Ticarcilline & $\mathrm{R}$ & $\geq 128$ & Pipéracilline & I & $=32$ \\
\hline $\begin{array}{l}\text { Piperacilline/ } \\
\text { Tazobactam }\end{array}$ & $\mathrm{R}$ & $\geq 128$ & Céfuroxime & $\mathrm{R}$ & $\geq 64$ \\
\hline Cefalotine & $\mathrm{R}$ & $\geq 64$ & Céfuroxime axétil & $\mathrm{R}$ & $\geq 64$ \\
\hline Cefoxitine & $\mathrm{R}$ & $\geq 64$ & Céfixime & $\mathrm{R}$ & $\geq 4$ \\
\hline Cefotaxime & $\mathrm{R}$ & $\geq 64$ & Céftriaxone & $\mathrm{R}$ & $\geq 64$ \\
\hline Ceftazidime & $\mathrm{R}$ & $\geq 64$ & Céfépime & $\mathrm{R}$ & $=32$ \\
\hline Imipeneme & $\mathrm{R}$ & $\geq 16$ & Aztréonam & $\mathrm{R}$ & $\geq 64$ \\
\hline Ertapénème & NA & NA & & & \\
\hline Amikacine & $\mathrm{R}$ & $\geq 64$ & Méropénème & $\mathrm{R}$ & $\geq 16$ \\
\hline Gentamicine & $\mathrm{R}$ & $\geq 16$ & Lévofloxacine & $\mathrm{R}$ & $\geq 8$ \\
\hline Tobramycine & $\mathrm{R}$ & $\geq 16$ & Moxifloxacine & $\mathrm{R}$ & $\geq 4$ \\
\hline & & & Minocycline & $\mathrm{S}$ & $\leq 1$ \\
\hline Acide nalidixique & $\mathrm{R}$ & $\geq 32$ & Tétracycline & $\mathrm{S}$ & $=4$ \\
\hline Ciprofloxacine & $\mathrm{R}$ & $\geq 4$ & Tigécycline & $\mathrm{S}$ & $\leq 0,5$ \\
\hline Ofloxacine & $\mathrm{R}$ & $\geq 8$ & Chloramphénicol & $\mathrm{S}$ & $=4$ \\
\hline Nitrofurantoine & $\mathrm{R}$ & $\geq 512$ & Triméthoprime & $\mathrm{R}$ & $\geq 160$ \\
\hline Triméthoprime/ & $\mathrm{R}$ & $\geq 320$ & Colistine & NA & NA \\
\hline Sulfaméthoxazole & & & Ticarcilline/Ac. & NA & NA \\
\hline
\end{tabular}

NA: Non appliqué (concerne les antibiotiques non requis pour ce germe et dont les résultats sont supprimés par le système Vitek 2 Compact 15); I: intermédiaire; R: résistance; S: sensibilité

Tableau II : Résultats des examens hématologiques et biochimiques chez la patiente

\begin{tabular}{ccc}
\hline & Hémogramme & \\
\hline $\begin{array}{c}\text { Paramètres } \\
\text { hématologiques }\end{array}$ & $\begin{array}{c}\text { Résultats } \\
\text { obtenus }\end{array}$ & $\begin{array}{c}\text { Valeurs } \\
\text { de référence }\end{array}$ \\
\hline Hémoglobine & 12,1 & $12-17 \mathrm{~g} / \mathrm{dL}$ \\
Hématocrite & 42,9 & $37-47 \%$ \\
Hématies & 4,59 & $(4,2-5,2) \times 10^{12} / \mathrm{L}$ \\
Globules blancs & 4,9 & $(4-10) \times 10^{9} / \mathrm{L}$ \\
& & \\
Granulocytes & 1,2 & $(2-7) \times 10^{6} / \mathrm{mm}^{3}$ \\
Lymphocytes & 3,2 & $(0,8-4) \times 10^{6} / \mathrm{mm}^{3}$ \\
Monocytes & 0,5 & $(0,1-1,2) \times 10^{6} / \mathrm{mm}^{3}$ \\
VGM & 93,6 & $(80-100) \mathrm{fL} \mathrm{ou} \mu \mathrm{m}^{3}$ \\
CCMH & 282 & $(320-360) \mathrm{g} / \mathrm{L}$ \\
TCMH & 26,3 & Pg/hématie \\
Plaquettes & 319 & $(100-300) \times 10^{3} / \mathrm{mm}^{3}$ \\
(VS) 1ère heure & $66 \mathrm{~mm}$ & $(3-8) \mathrm{mm}$ \\
\hline
\end{tabular}

\begin{tabular}{ccc}
\hline & Examens biochimiques & \\
\hline Paramètres & Résultats de l'analyse & Valeurs de références \\
\hline GOT/AST & 24 & $\leq 31 \mathrm{U} / \mathrm{L}$ \\
GPT/ALT & 30 & $\leq 32 \mathrm{U} / \mathrm{L}$ \\
Bilirubine totale & 20,7 & $\leq 18,8 \mu \mathrm{mol} / \mathrm{L}$ \\
Bilirubine directe & 6,9 & $\leq 4,3 \mu \mathrm{mol} / \mathrm{L}$ \\
\hline
\end{tabular}

VGM: volume globulaire moyen des hématies; CCMH: concentration corpusculaire moyenne en hémoglobine ; TCMH : teneur corpusculaire moyenne en hémoglobine VS: vitesse de sédimentation; GPT: Transaminases glutamique pyruvique ou ALAT: Alanine aminotransférases; GOT: Transaminase glutamique oxaloacétique ou ASAT: Aspartate aminotransférase 